\title{
GESTIÓN DIRECTIVA EN ESTABLECIMIENTOS DE EDUCACIÓN MEDIA TÉCNICO PROFESIONAL: DESAFÍOS PARA EL LIDERAZGO
}

\section{Leandro Sepúlveda ${ }^{1}$ y María José Valdebenito}

\begin{abstract}
RESUMEN
Este artículo aborda el análisis de las modalidades de gestión institucional y las prácticas de liderazgo directivo en establecimientos que imparten educación media técnico profesional (EMTP) en Chile. Para tal efecto, se revisan las estrategias de orientación vocacional existentes en estos centros, los procesos de articulación entre la formación general y la formación diferenciada, y las acciones de vinculación con actores productivos para el apoyo de los procesos de formación para el trabajo. A partir de una metodología de estudio de casos, se evidencia un predominio de formas de liderazgo que asumen un carácter delegativo, observándose una débil capacidad de los equipos directivos por asumir las tareas que son propias de las respectivas especialidades de la EMTP. Las exigencias externas para el aseguramiento de la calidad educativa concentran parte importante de los esfuerzos de dirección de estos establecimientos, relegando a un plano secundario los objetivos de formación profesional y dificultando las tareas de vinculación con empresas u otros actores productivos locales o regionales. Como se desprende de algunas experiencias innovadoras, la promoción de un liderazgo que favorezca acciones de intermediación intra e interestablecimientos educacionales, constituye una alternativa de particular interés para el desarrollo de la EMTP y sus desafíos futuros.
\end{abstract}

Conceptos clave: educación media técnico profesional, gestión escolar, liderazgo escolar.

\section{SCHOOL MANAGMENT IN TECHNICAL AND VOCATIONAL EDUCATION: CHALLENGES FOR LEADERSHIP}

\section{ABSTRACT}

This article addresses the analysis of the institutional management modalities and the management leadership practices in educational institutions offering secondary TVET education in Chile. To this end, the vocational orientation existing in these centers are reviewed, along with the articulation processes between the general and specialized education and the actions aimed to promote links with multiple industries and relevant actors with the goal of supporting education-to-work processes. The case study conducted, revealed a predominance of leadership styles that adopt a delegative nature, with little capacity of the managing teams to take on tasks that are specific of the different secondary TVET areas of specialization. The external requirements for education quality assurance demand a significant portion of these institutions' management efforts, relegating to a second level the professional training goals and making it

1 Universidad Alberto Hurtado, Santiago, Chile. Contacto: lesepulv@uahurtado.cl

2 Universidad Alberto Hurtado, Santiago, Chile. Contacto:mavaldeb@uahurtado.cl 
more difficult to develop links with companies or other production actors, local or regional. As can be inferred from some innovative experiences, promoting a leadership model that favors intra and inter mediation actions of educational institutions, is an alternative of particular interest for the development of secondary TVET and its future challenges.

Key concepts: school leadership; school management, technical and vocational education and training.

\section{Introducción}

Las características particulares que asume la gestión directiva al interior de establecimientos que imparten educación media técnico profesional (EMTP) en Chile constituye un tema débilmente abordado por la investigación educativa en general, y los estudios acerca de liderazgo en particular. Pese a la relevancia de la EMTP en términos de la cobertura que alcanza su matrícula en los niveles de $3^{\circ}$ y $4^{\circ}$ año medio, existe un muy bajo nivel de evidencia empírica acerca de las formas organizativas, roles directivos, dinámicas intraestablecimientos y estrategias destinadas a lograr un vínculo con el entorno productivo para el cumplimiento de los objetivos curriculares de esta alternativa de formación de la enseñanza media. No obstante que en años recientes ha habido una mayor preocupación por el diagnóstico de los principales problemas que afectan la provisión de la educación técnica y, muy particularmente, las dificultades de adecuación curricular a los requerimientos predominantes en el mercado laboral y las demandas socioproductivas (Comisión para la Educación de Calidad para Todos, 2016; Organización para la Cooperación y el Desarrollo Económicos, OCDE, 2017), en general, se ha brindado poca atención a lo que ocurre al interior de los propios establecimientos educacionales y existe una muy baja consideración en torno a la complejidad que implica la implementación de los procesos de gestión educativa para directivos y docentes en este tipo de instituciones.

En efecto, la gestión de la EMTP no solo resulta compleja debido a la demanda específica que se hace a esta alternativa de formación para dar respuesta a las necesidades del mercado laboral y favorecer la transición escuela-trabajo de los jóvenes que estudian alguna de sus especialidades, sino que también por cuestiones propiamente 
socioeducativas; entre otras: el doble objetivo de formación para el trabajo y la continuidad de estudios que sostiene su propuesta curricular; la complejidad de la organización interna y el desafío de alcanzar una colaboración pedagógica entre profesionales de diversas áreas de especialización; y la implementación de un modelo formativo de carácter diferenciado —un currículo basado en competencias, bajo una modalidad de organización modular - sobre el que no existen criterios transversales de evaluación o medición de su calidad (Sepúlveda, 2016). Estos y otros factores hacen de los establecimientos de la EMTP una realidad particular dentro del sistema educativo en su conjunto, por lo que el análisis de las formas de gestión institucional prevalecientes exige poner atención en aspectos que tradicionalmente no son considerados en la discusión en torno al liderazgo educativo.

En este artículo se propone el análisis de tres ámbitos críticos de la provisión educativa de la EMTP, donde el liderazgo directivo juega o puede jugar un rol crucial:

- el ejercicio del doble rol de formación para el trabajo y continuidad de estudios, y las acciones de diagnóstico y orientación vocacional de sus estudiantes;

- los procesos de enseñanza y aprendizaje al interior de esta alternativa curricular, donde la articulación entre la formación general y la formación propia de cada especialidad es particularmente relevante; y

- la vinculación con el entorno y, de manera específica, la capacidad institucional para generar lazos con una red de empresas y actores productivos relacionados con las especialidades impartidas en los respectivos establecimientos.

Acerca del primer ámbito, estudios recientes destacan la tensión asociada al doble propósito de formación para el trabajo y continuidad de estudios de sus egresados, más aún en el marco de la masificación de la educación superior y el incremento de las aspiraciones de los jóvenes por continuar estudios una vez finalizada su enseñanza secundaria (Sepúlveda y Valdebenito, 2014). Esta situación implica nuevas exigencias para la conducción de los procesos que garanticen la calidad y pertinencia de la oferta educativa, así como también, la 
necesidad de minimizar el efecto negativo que pueda implicar la canalización temprana de una opción vocacional de los estudiantes (Agencia de Calidad de la Educación, ACE, 2016a).

A nivel de las prácticas de liderazgo relativas a los procesos de enseñanza y aprendizaje, los diagnósticos son recurrentes en señalar la necesidad de contar con modalidades de gestión pedagógica que promuevan la articulación e integración de ambas perspectivas formativas, así como dinámicas conducentes al desarrollo de dispositivos que guíen a los jóvenes en la búsqueda de sus proyectos personales de manera integral (Sevilla y Sepúlveda, 2016).

Finalmente, en lo que respecta a la vinculación con el entorno, los equipos directivos de este tipo de centros educativos enfrentan desafíos exigentes en su relación con el medio. La actualización e implementación de las especialidades, la demanda por espacios adecuados para la realización de prácticas profesionales, el desarrollo de mecanismos de articulación con entidades de educación superior y actores productivos, que cumplan con los requerimientos definidos en el currículo de las distintas especialidades, son algunos de los puntos centrales que se destacan en este ámbito (Comisión Nacional para la Productividad, 2018).

En este marco resulta pertinente indagar acerca de las formas organizativas y la modalidad de gestión existente en los establecimientos de la EMTP, a fin de responder a estos desafíos $y$, en un sentido más general, analizar el rol y las funciones que abordan los equipos directivos en esta tarea. Este es el foco de interés del presente artículo. Para tal efecto, el análisis de las modalidades de gestión y liderazgo directivo se realiza a partir de la evidencia empírica levantada en un estudio de casos de establecimientos que imparten EMTP en tres regiones de Chile.

\section{Antecedentes generales}

La EMTP representa una alternativa de formación que cubre un porcentaje relevante de los estudiantes que cursan los dos últimos años de la enseñanza secundaria en Chile. En la actualidad, cerca de 
165.000 jóvenes estudian alguna de las especialidades reconocidas por el currículo vigente en los más de 950 establecimientos educacionales que imparten esta formación a lo largo del país. Hacia el año 2016, la EMTP alcanzaba una cobertura del 38\% de los estudiantes de $3^{\circ} \mathrm{y}$ $4^{\circ}$ año medio (ACE, 2016b) ${ }^{3}$.

La actual oferta EMTP surgió de la reforma educacional implementada durante la década de los noventa, cuando se estableció una modalidad de educación diferenciada para los dos últimos años de la enseñanza obligatoria, la cual estaba destinada a desarrollar competencias para el trabajo en los estudiantes, a través de una formación que entregara herramientas adecuadas para la autonomía laboral y, muy particularmente, que permitiera enfrentar los cambios en la estructura del empleo y las transformaciones tecnológicas que caracterizan los tiempos actuales (Sevilla, 2011).

A partir de esta reforma curricular, la formación diferenciada se ha concebido como un modelo de educación profesional de carácter modular basado en un enfoque de competencias; su objetivo es proporcionar una formación teórica y práctica que permita al estudiante el manejo de los aspectos más relevantes de la especialidad elegida ${ }^{4}$. En las bases curriculares vigentes para la formación diferenciada técnico profesional de la educación media, se ha definido un contexto laboral y un conjunto de objetivos de aprendizaje para cada especialidad, los que deben ser logrados al final de los dos años de formación diferenciada. Estos objetivos configuran el perfil de egreso

3 Proporcionalmente representa una de las tasas más altas de participación en la matrícula de educación secundaria en América Latina (Sevilla, 2017). Se trata, además, de un modelo formativo dirigido a estudiantes de las familias más pobres de la sociedad. El 64\% de la población estudiantil de la ESTP corresponde a jóvenes pertenecientes a familias de los dos deciles de ingresos más bajos y el 66,4\% de los liceos que imparten educación técnica se encuentran en el quintil de mayor vulnerabilidad.

4 El actual marco curricular reconoce la existencia de 15 sectores productivos, 35 especialidades y 17 menciones al interior de estas. Por cierto, la necesidad de adecuación de la oferta formativa es un requerimiento constante para una educación técnica que se construye (o debería construirse) en función de los requerimientos productivos y la oferta laboral en el país. La elección de la especialidad a estudiar se realiza al finalizar el $2^{\circ}$ año de la enseñanza media y, como se verá, constituye uno de los nudos críticos de la ESTP y que interroga sobre alternativas de liderazgo institucional para favorecer una opción informada para el estudiante y su entorno familiar. Véase http://www.tecnicoprofesional.mineduc.cl/wpcontent/uploads/2016/03/Bases-formacion-Descripci\%C3\%B3n-de-las-Especialidades.pdf 
de los estudiantes, y en ellos se explicita lo mínimo y fundamental que debe aprenderse, que incluye las competencias técnicas propias de la especialidad o de la mención, así como también los objetivos de aprendizaje genéricos que son comunes a todas las especialidades, y que remiten a competencias transversales, independientemente del sector económico de cada programa formativo (Ministerio de Educación de Chile, Mineduc, 2015; Miranda, 2005).

Aunque el actual modelo curricular de la EMTP parece responder adecuadamente a las características de un moderno sistema de provisión educativa de nivel técnico (Organización de las Naciones Unidas para la Educación, la Ciencia y la Cultura, Unesco, 2016), el diagnóstico acerca de su implementación, empero, evidencia un conjunto de problemas. El informe de la OCDE sobre la educación en Chile (2017) indica que, en este sector formativo, la inexistencia de instrumentos de evaluación y supervisión dificulta que las instituciones desarrollen propuestas funcionales a los requerimientos del mundo productivo. Por otra parte, uno de los aspectos más problemáticos respecto de este sistema educativo remite a las limitaciones existentes para un análisis en torno a la calidad de la EMTP. En breve, este hecho se debe a la falta de mecanismos externos de evaluación de aprendizajes o de adquisición de competencias de los estudiantes que cursan estudios en las distintas especialidades formativas (Arias, Farías, González-Velosa, Huneeus y Rucci, 2015).

Desde una mirada global, el reporte de la Comisión Externa de Formación Técnica para el Ministerio de Educación (2009) señaló tempranamente las grandes debilidades institucionales para alcanzar una formación de calidad, debido a la escasez de recursos materiales e insumos para el trabajo práctico en los establecimientos y a la desvinculación que existe entre este modelo formativo, la oferta de educación técnica de nivel superior y el mundo productivo. Este diagnóstico de la EMTP continúa vigente hasta ahora (véase, por ejemplo, Comisión Nacional para la Productividad, 2018; Educación 2020, 2016), evidenciándose las dificultades de innovación en las políticas para este sector y, muy particularmente, la ausencia de una institucionalidad fuerte, capaz de hacer frente a los principales problemas detectados (Mineduc, 2018). 
Los antecedentes que entregan estos estudios en cuanto a la EMTP, tanto en lo referido a la organización formativa como a la experiencia y trayectoria de sus estudiantes, ha planteado la necesidad de una discusión profunda acerca del carácter de esta modalidad formativa y las reformas que eventualmente requerirá hacia el futuro. De igual manera, los importantes cambios en la situación de posegreso de los estudiantes (Larrañaga, Cabezas y Dusaillant, 2011) interrogan acerca de la pertinencia de sostener una modalidad de formación vocacional a nivel de la enseñanza media (o por lo menos, introducir mecanismos de mayor flexibilización), teniendo en consideración el crecimiento sostenido de la matrícula de los jóvenes que egresan de esta modalidad formativa en el nivel de la educación postsecundaria.

Con todo, una discusión estratégica acerca de la situación y proyección de la EMTP hacia el futuro requiere un mayor acopio de antecedentes e investigaciones que incluyan campos hasta ahora débilmente abordados. En este marco, como se ha indicado, un ámbito que ha sido marginal en la investigación educativa (como también débilmente considerado por las propias políticas sectoriales) dice relación con la complejidad que implica gestionar establecimientos educativos de carácter técnico profesional, los que deben cumplir con el conjunto de exigencias y requerimientos de cualquier otro centro educativo tradicional, pero que también tienen que sumar el conjunto de exigencias que son propias de la formación para el trabajo.

Pese a la existencia de algunos estudios pioneros respecto de esta temática realizados a comienzos de la década del noventa (por ejemplo, Cariola, 1992; Del Campo y Cárcamo, 1993), llama la atención que la investigación acerca de la gestión institucional y el liderazgo educativo en la educación técnica ha sido particularmente escasa, centrando su atención fundamentalmente en aspectos relativos a la identificación y el desarrollo de capacidades en los distintos actores (directores, docentes, estudiantes) con un énfasis prescriptivo, sin considerar la especificidad de la misma y desestimando interrogantes que buscan dar cuenta de las prácticas, relaciones y dinámicas propias relativas al liderazgo educativo en el marco de las exigencias de un modelo de formación diferenciado (Carbone, Fuenzalida y Farías, 2017). El grueso de la producción académica 
ha enfatizado en cuestiones que tienen que ver con la estructura y el diseño curricular (por ejemplo, Cariola, Labarca, Irigoin, Erazo y Fox, 1994; Donoso y Corvalán, 2012), pero con una débil consideración de la experiencia institucional en concreto, donde tales estructuras se ponen en juego. Dada la relevancia de esta temática, este artículo tiene por objetivo, a partir de un estudio de casos, el análisis de las prácticas y dinámicas asociadas al liderazgo escolar en la EMTP, identificando las dificultades, desafíos actuales y escenarios futuros en la provisión educativa y articulación de la demanda socioproductiva que enmarca su desarrollo.

\section{Perspectiva de análisis}

El sello fundamental que caracteriza a la educación técnico profesional y que la distingue de la educación general es su vínculo con el mundo del trabajo, lo que implica que esta modalidad formativa debe cumplir con, al menos, un doble propósito: por una parte, desarrollar acciones destinadas a mejorar el bienestar social y económico de los individuos $y$, por otra, aportar a la formación de la fuerza de trabajo necesaria para el mercado laboral y los esfuerzos productivos de toda sociedad (Adams \& Gamage, 2008). Como consecuencia, la educación técnico profesional que se entrega en el nivel educativo formal requiere una estructura organizativa comparativamente más compleja, incluyendo un conjunto de procedimientos y condiciones necesarias para la formación destinada a la vida laboral (Özdemir \& Şahin, 2016).

Junto con lo anterior, no puede desconocerse que el proceso de modernización económica verificado en las últimas décadas ha supuesto nuevas condiciones y retos a este sistema formativo. A nivel mundial, las rápidas transformaciones del sistema productivo han llevado a que esta modalidad de estudios se vea enfrentada a la necesidad de sucesivos cambios e innovaciones. Esto se expresa, por una parte, en la adopción de una progresiva flexibilidad en términos de sus contenidos curriculares, capacitación y experiencia docente (Park \& Jeong, 2013) y, por otra, en el enfrentamiento de condiciones cada vez más competitivas para su financiamiento y el logro de objetivos de autoeficiencia institucional (Simons \& Harris, 2014). A la educación técnico profesional se le plantea el desafío imperativo 
de responder a los rápidos y continuos cambios en la tecnología, el ambiente económico y la naturaleza del trabajo y, en consecuencia, desarrollar la capacidad de formar trabajadores profesionales, flexibles, con competencias necesarias para las nuevas demandas laborales y, por tanto, preparados para hacer frente al aprendizaje a lo largo de la vida (Boateng, 2012; Mulder, Messmann y König, 2015).

Ante este conjunto de demandas, uno de los elementos clave para el desarrollo del quehacer de las instituciones que imparten formación técnico profesional remite al liderazgo de sus organizaciones y a la forma en que este se expresa en la construcción y sostén de redes o vínculos que estos establecen con su entorno inmediato. Esta característica es destacada como una condición necesaria para posibilitar la adaptabilidad a las nuevas necesidades de los sistemas educativos y el mercado laboral (Khatajabor, Asnuldahar, Sadaadamu \& Buntat, 2012).

Aunque en la literatura actual acerca de la educación y la formación para el trabajo predomina un tipo de análisis que remite a modalidades formativas y a formas organizacionales específicas - que no siempre considera los desafíos propios de un modelo de educación formal obligatorio - se considera que, de manera transversal, todo centro educativo que incluya la educación para la vida laboral debería cumplir con los requerimientos de adecuación de sus propuestas educativas a las demandas presentes y futuras de competencias para el trabajo (Amaral, De Diego, Pagés y Prada, 2018).

En este marco, existe una amplia coincidencia en señalar que los principales desafíos de los centros de educación técnica en la actualidad —independientemente de su lugar en la institucionalidad educativa - se relacionan con los siguientes aspectos:

- una adecuada conexión de la oferta formativa con el mercado laboral y la formación de capital humano;

- un énfasis en el trabajo en red con distintos actores de la comunidad educativa y laboral (organizaciones sindicales, asociaciones sectoriales y profesionales y empresas) para adecuar las propuestas formativas a la realidad circundante; 
- una perspectiva de innovación permanente y la necesidad de cambiar continuamente los contenidos de aprendizaje y metodologías de trabajo de acuerdo con las señales que entrega el mercado laboral;

- énfasis en un rol de responsabilidad social por la inclusión, debido a que se trata de un modelo formativo destinado a una población con menores alternativas de elección académica; y

- socialización y difusión de la oferta de formación disponible en cada centro educativo (European Centre for the Development of Vocational Training, Cedefop 5 , 2011).

De todo lo anterior, es el vínculo con el mercado del trabajo y la adecuación de la oferta formativa a la demanda productiva la variable más importante en la gestión de este tipo de centros.

En efecto, el tipo de relación entre la educación y el trabajo constituye una de las características clave que determinaría la calidad de la formación técnica de un sistema educativo (Mulder et al., 2015), siendo el principal elemento distintivo del liderazgo directivo en la educación técnico profesional. Este vínculo otorga el marco de acciones generales para cada uno de los siguientes factores, así como también las habilidades de liderazgo que cada uno de estos demanda a quienes desempeñan tareas directivas en contextos técnico profesionales. La relación entre educación y trabajo constituye un elemento transversal de la formación técnico profesional, pero las formas en que esta se despliega varían de acuerdo con las lógicas de cada país y obedecen a contextos más amplios como las políticas de bienestar, el grado de centralización de los países, los marcos institucionales de la educación y los mecanismos de regulación de los mercados laborales, entre otros. De acuerdo con Mulder et al. (2015) para comprender la naturaleza de la relación entre educación y trabajo es fundamental la consideración de los contextos históricoculturales, políticos e institucionales de cada sistema de formación en particular.

5 Centro Europeo para el Desarrollo de la Formación Profesional, Unión Europea. Este documento sintetiza los principales esfuerzos de coordinación de la oferta actual de la educación y formación para el trabajo a nivel europeo y que incide de manera significativa en las orientaciones de política para los centros de formación en diferentes lugares del mundo. 
Raffe (2007), por su parte, distingue dos tipos generales de asociación entre el trabajo y la educación técnico profesional: aquellos sistemas con alianzas fuertes denominados modelos con lógica de empleo y aquellos con alianzas débiles, denominados con lógica educativa. Los sistemas donde predomina una lógica de empleo son aquellos donde los programas y las estructuras institucionales del sistema educacional técnico profesional reflejan, de manera determinante, las necesidades de los empleadores y el mercado laboral. Por el contrario, los sistemas donde predomina una lógica educativa, el vínculo con los empleadores es más tenue y tienden a tener menos diferencias con el sistema de educación general (Raffe, 2007). Los sistemas de educación técnico profesional de Alemania, Dinamarca y los Países Bajos han sido tradicionalmente de lógica de empleo, en cambio en países como Australia e Inglaterra ha predominado la lógica educativa (Polesel, Klatt, Blake \& Starr, 2017).

El caso chileno, desde este punto de vista, se acerca mayormente al modelo con lógica educativa, más aun considerando la integración curricular de la formación diferenciada dentro del sistema escolar formal. Pese al reconocimiento transversal de la importancia de la educación técnico profesional en el nuevo contexto de la economía global y el relativo acuerdo de los organismos internacionales respecto del rol clave que el liderazgo desempeña en la mejora de esta modalidad de estudio, la articulación entre las dimensiones de educación general y la formación para el trabajo representa un tema de difícil asunción. Esta falta de atención a los desafíos específicos que enfrentan los líderes de la educación técnico profesional puede provenir de la creencia de que el liderazgo en esta formación no difiere del liderazgo de la educación general y que, por lo tanto, no requiere una atención especial. Contrariamente a este supuesto, el presente artículo intenta relevar los aspectos específicos a los cuales se enfrentan los directivos y otros líderes dentro de las instituciones educativas técnico profesionales.

\section{Consideraciones metodológicas}

El material que sirve de base para este artículo tiene su origen en una investigación que desarrolló una estrategia de indagación de tipo 
cualitativa sobre la base de un estudio de $\operatorname{casos}^{6}$. El trabajo se centró en recoger antecedentes acerca de las prácticas de gestión existentes al interior de establecimientos de modalidad técnico profesional, así como las racionalidades que orientan el quehacer de los equipos directivos en los establecimientos educacionales EMTP y las formas de liderazgo existentes en su interior ${ }^{7}$.

El proceso de investigación se focalizó en el análisis de las experiencias y percepciones de los sujetos, teniendo en consideración la forma en que se desarrolla la actividad educativa en los liceos EMTP y las prácticas y dinámicas de liderazgo y organización institucional para hacer frente a los nudos de gestión reseñados en la sección precedente de este artículo. Para tal efecto, se prestó particular atención a la descripción de procesos y formas de dirección institucional para un adecuado cumplimiento de la formación diferenciada en los dos últimos niveles de la enseñanza media.

El estudio contempló la selección de una muestra intencionada de centros educativos que imparten EMTP en tres regiones del país: la Región Metropolitana, la de Valparaíso y la de La Araucanía. El criterio para la elección de las regiones donde se ubican los centros educativos combina representatividad (más del 50\% del total de la matrícula total del país) y diversidad (particularmente en el caso del establecimiento de tipo rural). Aunque originalmente la indagación estaba prevista considerando una muestra de seis establecimientos EMTP, finalmente

6 "El estudio de casos es el estudio de la particularidad y de la complejidad de un caso singular, para llegar a comprender su actividad en circunstancias importantes (...) el investigador cualitativo destaca las diferencias sutiles, la secuencia de los acontecimientos en su contexto, la globalidad de las situaciones personales" (Stake, 2010, p. 11).

7 La noción de racionalidad que utilizamos aquí refiere al control reflexivo que tienen los agentes sociales sobre su conducta y condicionantes sociales; pero a diferencia de un modelo que enfatiza en el condicionamiento estructural que hace de la racionalidad de la acción de los sujetos un ejercicio de ponderación entre oportunidades y constricciones que imponen las situaciones (como ocurre en la propuesta de Goldthorpe), el énfasis analítico en nuestro trabajo se acerca a la propuesta de la sociología de los desafíos sociales, que "son retos históricos, socialmente producidos, culturalmente representados, desigualmente distribuidos, que los individuos están obligados a afrontar en el seno de un proceso estructural de individuación” (Martuccelli y Santiago, 2017, p. 84). El objetivo de la sociología de los desafíos sociales es "proponer una problematización de las estructuras sociales a escala —y desde las experiencias— de los individuos. Es, en definitiva, partiendo de lo que vive el actor, y cómo lo experimenta, como se da cuenta de los grandes desafíos estructurales e históricos de una sociedad" (Martuccelli y Santiago, 2017, p. 93). 
el estudio se organizó en una indagación a cinco centros educacionales y un sistema de provisión educativa de carácter comunal que incluye tres establecimientos educativos en su interior ${ }^{8}$. La Tabla 1 resume el perfil de los centros seleccionados para el estudio. La selección cubrió tres variables relevantes que dan cuenta del sistema EMTP:

- el conjunto de alternativas de dependencia administrativa de los establecimientos de la EMTP en Chile, esto es, liceos municipales, particular subvencionados y de administración delegada";

- las principales tendencias en la modalidad de implementación, esto es, liceos que solo brindan enseñanza media y liceos que también imparten educación básica, además de un caso de liceo polivalente (que ofrece la alternativa científico humanista y técnico profesional a la vez ${ }^{10}$ ) y un liceo agrícola ${ }^{11}$; y

- tamaño de la población escolar y diversidad en la oferta de especialidades EMTP (de una a cuatro especialidades impartidas).

Tabla 1

Casos de estudio y principales características

\begin{tabular}{lll}
\hline Casos & Dependencia administrativa & Característica \\
\hline Caso 1 & Municipal & Solo educación media, cinco especialidades. \\
\hline Caso 2 & Administración delegada & Solo educación media, seis especialidades. \\
\hline Caso 3 & Particular subvencionado & Solo educación media, dos especialidades. \\
\hline Caso 4 & Particular subvencionado & Rural e internado, una especialidad. \\
\hline Caso 5 & Particular subvencionado & Polivalente, una especialidad. \\
\hline Caso 6 & Tres liceos municipales & $\begin{array}{l}\text { Educación básica y media; cuatro } \\
\text { especialidades, dos y dos. }\end{array}$ \\
\hline
\end{tabular}

Fuente: Elaboración propia.

8 Se trata de una experiencia donde la administración municipal juega un rol importante en la gestión y liderazgo de la provisión educativa técnico profesional, por lo que en el momento de la implementación del estudio se optó por innovar en la propuesta metodológica inicial, a fin de lograr evidencia de este modelo poco común en la organización de la administración educativa comunal en Chile.

9 En Chile existen alrededor de 70 establecimientos educacionales que el Estado ha delegado su administración a entidades privadas sin fines de lucro a través de un sistema de financiamiento a la oferta. Todos estos brindan formación técnico profesional, equivalente al $12 \%$ del total de la matrícula de la ESTP (Centro de Estudios de Políticas y Prácticas en Educación, CEPPE, 2017).

10 El 38\% de los establecimientos que imparte EMTP en Chile es polivalente.

11 Los liceos agrícolas imparten las especialidades agropecuarias y forestales; pese a su potencial relevancia estratégica en el desarrollo económico, solo cubren al 8\% del total de la matrícula de la ESTP. 
Los casos fueron seleccionados a partir de antecedentes disponibles en estudios precedentes relacionados con la educación media técnico profesional y representan diversas experiencias de implementación formativa. En términos globales, esta investigación se guía por el principio de estudio de casos instrumentales (Stake, 2013); al profundizar en las experiencias en concreto, se persigue brindar una comprensión de un ámbito temático en particular, como es la que orienta el objetivo principal de investigación.

En el levantamiento de información, se aplicaron entrevistas al director(a) del establecimiento, al jefe de la Unidad Técnico Pedagógica o UTP ${ }^{12}$, al jefe técnico de EMTP o jefe de producción (donde existiese) $^{13}$, al encargado(s) de especialidad(es) ${ }^{14}$ y a sostenedores. Se realizaron 36 entrevistas en total. De manera paralela al levantamiento de información empírica, el equipo responsable del estudio realizó un trabajo de revisión de fuentes secundarias en torno a la temática de interés y a la construcción de un marco analítico de los resultados. El análisis del material recogido en el estudio siguió los procedimientos básicos del análisis de contenido. El procedimiento analítico tuvo un carácter flexible, y se utilizó el programa de apoyo computacional ATLAS.ti para una primera fase de ordenamiento del material, favoreciéndose en las etapas posteriores el trabajo analítico interpretativo con el fin de relevar los aspectos más relevantes que surgían del tratamiento del material, en función de los objetivos específicos que perseguía este estudio. En este contexto, se identificaron los significados, categorías y patrones de los discursos asociados a las prácticas, experiencias y estrategias institucionales relevadas por los entrevistados. Un primer paso en este ejercicio analítico apuntó al levantamiento inductivo de elementos emergentes comunes y también diferenciales en función a la temática de estudio. Se utilizó codificación abierta y un sistema de categorías temáticas, que permitió luego realizar un análisis relacional basado

12 El jefe de UTP es el docente directivo responsable inmediato de organizar, coordinar y supervisar el trabajo técnico pedagógico de los distintos organismos del establecimiento educacional.

13 Aunque no siempre está presente en los establecimientos ESTP, este cargo representa la función de coordinación técnico pedagógica (y administrativa) de la formación diferenciada.

14 Docentes a cargo de la formación específica de las respectivas especialidades al interior del centro educativo. 
en las categorías comunes y diferenciadas más relevantes para esta investigación. En un segundo paso se procedió, de forma creciente, a la articulación de los componentes, buscando su coherencia interna e integración conceptual, con el objetivo de articular uno o varios modelos de estrategias de gestión institucional al interior de los establecimientos que constituían los casos de estudio.

Los hallazgos que se entregan a continuación representan una síntesis integrada de los principales resultados alcanzados en función de los ejes temáticos más relevantes o nudos críticos de la gestión de establecimientos de la EMTP ${ }^{15}$.

\section{Hallazgos del estudio}

Un primer aspecto que destaca de la revisión de los casos de estudio evidencia la heterogeneidad de la oferta formativa al interior de la EMTP. Debido a la complejidad del sistema y a la inexistencia de mecanismos de coordinación o regulación institucional (salvo, claro está, el currículo nacional), la provisión educativa técnico profesional se caracteriza por una multiplicidad de modelos de gestión, y una muy amplia gama de arquitectura organizacional destinada a la formación para el trabajo en el nivel de la enseñanza media. Pese a la diversidad de los casos revisados, es posible observar, con todo, algunas regularidades que dan cuenta de la manera en que se abordan los nudos críticos de este sistema formativo y los arreglos institucionales para su enfrentamiento. La Tabla 2 sintetiza los principales énfasis de gestión en cada caso de estudio:

15 Por una cuestión de espacio, no se incluyen en este trabajo citas textuales de los informantes, privilegiándose una síntesis analítica de los resultados en función de los ejes temáticos relevados. 
Tabla 2

Casos de estudio y sus principales características

\begin{tabular}{cl}
\hline Caso & \multicolumn{1}{c}{ Principales características } \\
\hline 1 & $\begin{array}{l}\text { Fuerte liderazgo dirección; innovación curricular en las especialidades } \\
\text { de la EMTP y estrategias de articulación con formación general (pasantías, }\end{array}$ \\
& colaboración docentes de ambas modalidades y figura de docentes pares); equipo \\
& ampliado de dirección (incluye coordinación TP dentro del equipo); orientación \\
& a educación comprensiva, incluyendo preparación para la PSU; vínculo con \\
& empresas a cargo de jefes de especialidad con apoyo de la Dirección.
\end{tabular}

2 Establecimiento líder en el área formativa y de alta valoración al interior de la EMTP; orientado a la formación para el trabajo y salida temprana a la vida laboral; conformación de grupos de especialidades en $1^{\circ}$ año medio evidencia que la elección vocacional es una decisión previa al ingreso a la institución; existencia de una red de empresas asociadas al sostenedor, figura de gerente como articulador del vínculo con el sistema productivo.

3 Equipo directivo amplio, incluye coordinador TP; inducción de estudiantes a especialidad en $2^{\circ}$ año medio, aunque también se utilizan criterios de asignación por notas; débil articulación formación general/especialidades; valoración liderazgo distribuido en la gestión directiva, aunque con limitaciones en el vínculo con empresas.

4 Establecimiento rural/internado. Especialidad es un rasgo de identidad institucional; fuerte vínculo comunitario; equipo consolidado (permanencia prolongada en cargos); débil vinculación entre la formación general/ especialidades y fuerte orientación hacia el perfil laboral de estudiantes; con todo, énfasis discursivo desde una lógica de inclusión social.

5 Establecimiento polivalente de orientación religiosa y compromiso con sectores pobres (discurso de inclusión social); tensión en la decisión de mantener oferta TP o transitar a una oferta general; preeminencia jefe especialidad en implementación formación diferenciada, débil articulación con formación general aunque disposición a avanzar en su mejoramiento; vínculos con empresas y otros apoyos vía corporación que ejerce como facilitador.

6 Sistema municipal; distintos énfasis de las tareas priorizadas en los tres establecimientos de acuerdo con el perfil y situación coyuntural; ejemplo de influencia externa para la definición de tareas prioritarias (ACE); rol destacado de jefes de UTP en tareas de articulación: liderazgo asociado a la movilización de los docentes (no siempre dispuestos a desarrollar las tareas); importante rol de intermediación con empresas por parte de coordinación TP de la Corporación Municipal.

Fuente: Elaboración propia.

La revisión de las experiencias sugiere que el sistema de EMTP en Chile, en términos generales, intenta responder a las exigencias de un modelo mayormente orientado a una lógica educativa, 
comprensiva ${ }^{16}$ o articulada. Solamente un caso (caso 2) da cuenta de un proyecto institucional que enfatiza en la formación para el trabajo, concibiendo la EMTP como un modelo terminal o, por lo menos, como un modelo que contiene un fin en sí mismo asociado a la formación laboral. Con todo, existe una muy débil evidencia de acciones que busquen hacer frente al desafío de una formación que favorezca la articulación. Solamente un caso de este estudio (caso 1) reporta acciones innovadoras en este campo, lo que incluye la colaboración de docentes de formación general (profesores de ciencias), que no tienen compromisos curriculares en los niveles de formación diferenciada, así como la implementación del trabajo colaborativo bajo un modelo de docentes pares y otras acciones impulsadas por el equipo directivo. En el resto de los casos, aunque se reconoce la necesidad de una mayor integración curricular, las acciones no pasan más allá de iniciativas de adaptación de algunas actividades de formación general en función de la especificidad de la o las especialidades impartidas.

La preeminencia de un sistema nacional de medición de la calidad de la educación que enfatiza en la focalización de logros en áreas tradicionales de la formación general y que no incluye mediciones específicas para la formación diferenciada, incide de manera relevante en esta situación. En la actualidad, el último nivel que incluye la medición que realiza el Sistema de Medición de Calidad de la Educación (Simce) corresponde al $2^{\circ}$ año de la enseñanza media y el único criterio que se ha incorporado en la evaluación de la EMTP es la tasa de titulación de estudiantes. Este hecho, y tal como lo demuestran de manera patente los establecimientos de dependencia municipal, incide en una mayor atención institucional a la formación

16 La distinción entre un modelo comprensivo y otro propiamente dirigido a la preparación para el trabajo en la enseñanza secundaria constituye un tema de debate de larga data. A comienzos del siglo XX, John Dewey criticó la existencia de dos modelos de formación diferenciados y su incidencia en la reproducción de lógicas de segmentación social (Stern, 2009); esta crítica no difiere mayormente de quienes observan en la actualidad el peligro de una reducción del modelo escolar a una oferta únicamente funcional a la demanda del sistema productivo y del mundo del trabajo (Maclean \& Pavlova, 2013). En este estudio hemos caracterizado el sistema ESTP chileno como orientado en lógica comprensiva, aunque se reconoce la ambigüedad estratégica que esto implica y que se evidencia en diferentes énfasis de las propuestas formativas que coexisten al interior del sistema (véase, Sepúlveda, Ugalde y Campos, 2011). 
impartida en el primer ciclo de la enseñanza media, relegando a un lugar secundario la formación diferenciada dentro de la estrategia de los respectivos equipos directivos.

Ahora bien, ¿cuáles son las principales tendencias observadas respecto de los nudos críticos identificados en este estudio?:

a. En relación con el proceso de adscripción a las especialidades

Uno de los ámbitos que presenta una menor injerencia institucional en la EMTP está asociado con el proceso de elección vocacional de los estudiantes y el seguimiento de sus opciones curriculares en el segundo ciclo de la enseñanza media. Aunque en la mayoría de los establecimientos se reportan acciones de información internas (ferias informativas para estudiantes de $2^{\circ}$ año medio, charlas de docentes de las especialidades o exposiciones de alumnos de los últimos años a sus compañeros de primer ciclo), estas resultan acotadas y bajo un marco de limitadas posibilidades de elección para los jóvenes. En efecto, por lo general la elección vocacional, cuando ocurre, es una decisión familiar y/o de los estudiantes, constreñida por la oferta disponible en los establecimientos cercanos a los lugares de habitación y, muchas veces, condicionada por las exigencias de equilibrio interno que deben asegurar los propios centros educativos. La mayoría de los establecimientos educacionales, pese a generar procesos de elección vocacional de sus estudiantes, introducen mecanismos de distribución de los mismos, donde cuestiones tales como la conducta previa o las calificaciones alcanzadas en los niveles de $1^{\circ}$ y $2^{\circ}$ año de enseñanza media, inciden en el acceso a una u otra alternativa curricular. El caso 2 da cuenta de una situación aún más compleja, en la medida en que la alta demanda de matrícula en el inicio de la enseñanza media, lleva a las autoridades de este centro educativo a organizar los cursos tempranamente en función de las diversas alternativas curriculares asociadas a las especialidades y que, en rigor, solo debería producirse a partir del $3^{\circ}$ año de enseñanza media. 
Tabla 3

Mecanismos de orientación/inducción vocacional en casos de estudio

\begin{tabular}{cl}
\hline Caso & \multicolumn{1}{c}{ Acciones de orientación } \\
\hline 1 & $\begin{array}{l}\text { Inducción a estudiantes en la oferta formativa; trabajo de equipos de } \\
\text { especialidades y orientación. Organización en función de criterios de } \\
\text { rendimiento escolar en casos específicos. }\end{array}$ \\
\hline 2 & $\begin{array}{l}\text { No existe. Elección previa al ingreso al establecimiento y conformación de } \\
\text { grupos en } 1^{\circ} \text { año medio. }\end{array}$ \\
\hline 3 & $\begin{array}{l}\text { Inducción a estudiantes en la oferta formativa. Organización en función de } \\
\text { criterios de rendimiento escolar. }\end{array}$ \\
\hline 4 & No existe. Liceo ofrece una única especialidad. \\
\hline 5 & $\begin{array}{l}\text { Polivalencia. Información acotada en } 2^{\circ} \text { año medio y mecanismos de } \\
\text { elección-asignación en función del rendimiento escolar. }\end{array}$ \\
\hline 6 & $\begin{array}{l}\text { Inducción a estudiantes en la oferta formativa. Organización en función de } \\
\text { criterios de rendimiento escolar en casos específicos. }\end{array}$ \\
\hline
\end{tabular}

Fuente: Elaboración propia.

Este nudo crítico de la gestión de la EMTP puede tener un efecto negativo en la propia dinámica institucional en la medida en que se producen desajustes de interés vocacional o, directamente, conductas de carácter instrumental por parte de los estudiantes, lo cual afecta la dinámica de organización interna, el vínculo con empresas sectoriales y las propias trayectorias educativo-laborales futuras de los jóvenes. En general, se observan capacidades débiles para hacer frente a este tema; incluso, en algunos casos, esto no se reconoce como un problema, que evidencia una lógica predominante basada en la elección individual y débiles soportes institucionales que permitan informar y orientar adecuadamente a los estudiantes en una decisión que resulta muy relevante para su proyección futura.

Las acciones institucionales de información acerca de las características de la oferta existente recaen fundamentalmente en los jefes y el equipo de docentes de cada especialidad; la amplitud e incidencia de esta estrategia depende, con todo, del ejercicio de ciertos liderazgos institucionales, que están concentrados generalmente en el jefe de formación TP (cuando este cargo existe), o bien, en los respectivos jefes de UTP. 
b. Respecto de la relación entre formación general y formación en especialidades

A diferencia del punto anterior, este aspecto sí es parte de la preocupación institucional. Muchos de los entrevistados mencionan el incremento de acciones correctivas o de innovación en la gestión pedagógica destinadas a hacer frente al desajuste que se produce entre la formación general y la formación en las respectivas especialidades, cuando se hace la diferenciación curricular entre educación media científico-humanista (EMCH) y EMTP. La tensión existente entre la provisión de educación general y especialidades ha sido destacada anteriormente, y se relaciona con cierta ambigüedad estratégica de nuestro modelo técnico profesional (Sepúlveda et al., 2011). En efecto, pese a que algunos discursos de agentes educativos tienden a enfatizar en la lógica del empleo, en un sentido más transversal, los establecimientos y sus directivos se encuentran condicionados por un sistema que se rige bajo una lógica educativa. En el caso 1 se señala que esto es así, porque existen cambios en las aspiraciones y expectativas de los estudiantes, quienes desean, mayoritariamente, continuar estudios una vez finalizada la enseñanza media. El predominio de esta lógica también se debe, como se reporta en el caso 6, a la organización del sistema: la ACE es vista como un organismo externo con capacidad de castigo y recompensa, que impone o sugiere modalidades de trabajo alternativo y obliga a dinamizar nuevos liderazgos internos para hacer frente a esta situación.

Tabla 4

Presencia de acciones de articulación curricular en casos de estudio

\begin{tabular}{ll}
\hline Caso & \multicolumn{1}{c}{ Nivel de articulación/preocupación institucional } \\
\hline 1 & Fuerte; preocupación por trayectorias educativo-laborales. \\
\hline 2 & Débil; mayormente orientado a formación para el trabajo. \\
\hline 3 & Mediano; aunque favorece articulación. \\
\hline 5 & Débil; mayormente orientado hacia la inclusión social. \\
\hline 6 & $\begin{array}{l}\text { Mediano; aunque creciente preocupación por trayectorias educativo- } \\
\text { laborales. }\end{array}$ \\
\hline
\end{tabular}

Fuente: Elaboración propia. 
De este modo, la articulación entre formación general y la formación diferenciada de la EMTP en la enseñanza media constituye un nudo relevante y en el cuál es posible observar no solo una preocupación al interior de los centros educativos, sino que crecientes iniciativas de intervención pedagógica. Acciones tales como el trabajo coordinado entre docentes de ambas modalidades, la adaptación curricular de la formación general a los módulos de la EMTP, la inclusión de contenidos de formación general no contemplados en el currículo TP, o la participación de docentes en clases o módulos de sus pares, son algunos ejemplos en esta dirección. No obstante, lo anterior no implica necesariamente que se trate de un proceso generalizado en este tipo de establecimientos, pero su enfrentamiento - como lo reportan los casos de estudio-, tiene implicancias en la forma de organización de los equipos directivos y, muy particularmente, en la emergencia de nuevas formas de liderazgo.

\section{c. Gestión de especialidades y relación con el mundo productivo}

En el análisis de casos fue posible corroborar que para el cumplimiento de esta tarea, el rol del encargado de la respectiva especialidad es fundamental. Su éxito depende de la amplitud y calidad de las redes de colaboración institucional, de su experiencia de trabajo previo y de los vínculos profesionales que haya establecido a lo largo de su carrera.

La individualización de esta función y la delegación de tal responsabilidad por parte del equipo de dirección de los establecimientos en una única persona, es una característica extendida en el sistema de la EMTP en Chile, y se refleja como evidencia en los casos 3 y 5 de esta investigación. Un aspecto más relevante aún es que, por lo general, estos profesionales no participan de la instancia de gestión directiva del centro educativo, por lo que muchas veces los problemas o necesidades asociadas a su gestión, se trabajan de manera parcelada o desvinculada del resto de las temáticas tradicionales al interior de los establecimientos. En este modelo, el director o directora puede jugar un rol de liderazgo importante en el quehacer pedagógico institucional, pero dedica una menor atención 
a lo propio de la formación diferenciada y, muy particularmente, a la red de relaciones que son necesarias para una adecuada provisión educativa de las especialidades que ofrece el centro. Este modelo se sintetiza en la Figura 1:

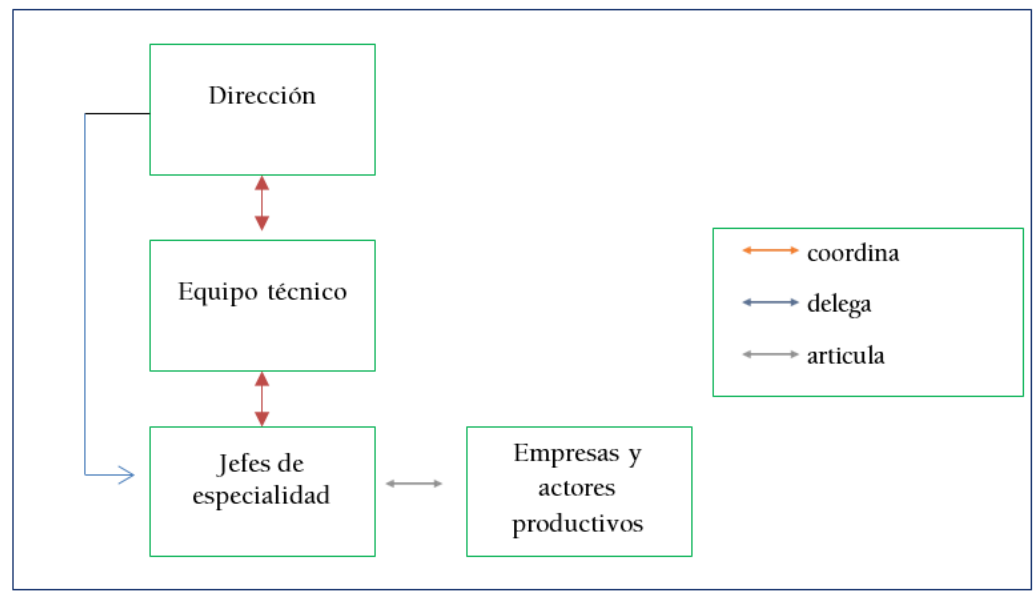

Figura 1. Modelo delegativo de la gestión directiva. Fuente: Elaboración propia.

Como se evidenció en la revisión bibliográfica, una buena conexión con el mercado laboral requiere de una planificación de la oferta educativa para que sea capaz de dar respuesta a las necesidades presentes y futuras del sector productivo que organiza la oferta de formación. La ausencia de mecanismos institucionales que favorezcan el seguimiento de la dinámica de este sector y su demanda laboral, sumada a la débil capacidad de analizar los cambios e innovaciones tecnológicas de la actividad productiva, son solo dos aspectos sobre los cuales este modelo organizativo — debido a la atomización de las tareas - , presenta poca capacidad de incidencia.

Un segundo modelo de gestión de la EMTP puede denominarse de articulación y apoyo y remite a un tipo de gestión directiva que tiene un mayor protagonismo en el proceso de vinculación con el mundo productivo y su consecuente revisión y reelaboración de las mallas curriculares y la oferta formativa a los estudiantes. En este estudio, este modelo está claramente representado por el caso 1 , no solo por el liderazgo directivo, sino también, porque su gestión 
tiene implicancias en el desarrollo de competencias pedagógicas de los docentes y en el impulso a la innovación en las prácticas de enseñanza-aprendizaje. No existe evidencia de esta modalidad de liderazgo en el resto de los casos en estudio; se trata más bien de una forma de gestión directiva altamente exigente, ya que combina capacidades de propuesta y conducción en el ámbito administrativo como en lo propiamente instruccional, aspecto particularmente débil para el conjunto de los actores del resto de las experiencias.

El desarrollo de este modelo sugiere la existencia de un tipo de liderazgo institucional que favorece procesos de innovación en la educación técnico profesional y formas de organización interna que operan como un círculo virtuoso, evitando que la oferta técnica profesional aparezca como una alternativa de segundo nivel, y relevando los aprendizajes y las disposiciones para la vida laboral de sus estudiantes. Tal como lo expresan Polesel et al. (2017), este tipo de establecimientos puede estrechar lazos con la industria local o con redes de actores del mundo productivo que observan en estos centros una realidad distintiva para la colaboración. El rol directivo se sustenta en una fuerte colaboración y coordinación institucional $y$, para posibilitar la tarea de vinculación con el medio, se requiere el ejercicio de un liderazgo distribuido con los participantes de su equipo técnico directivo. Los casos 1 y 5 son los que presentan una mayor densidad de funciones, al no reducir la coordinación técnico pedagógica o de organización de la oferta TP en un solo cargo en específico. 


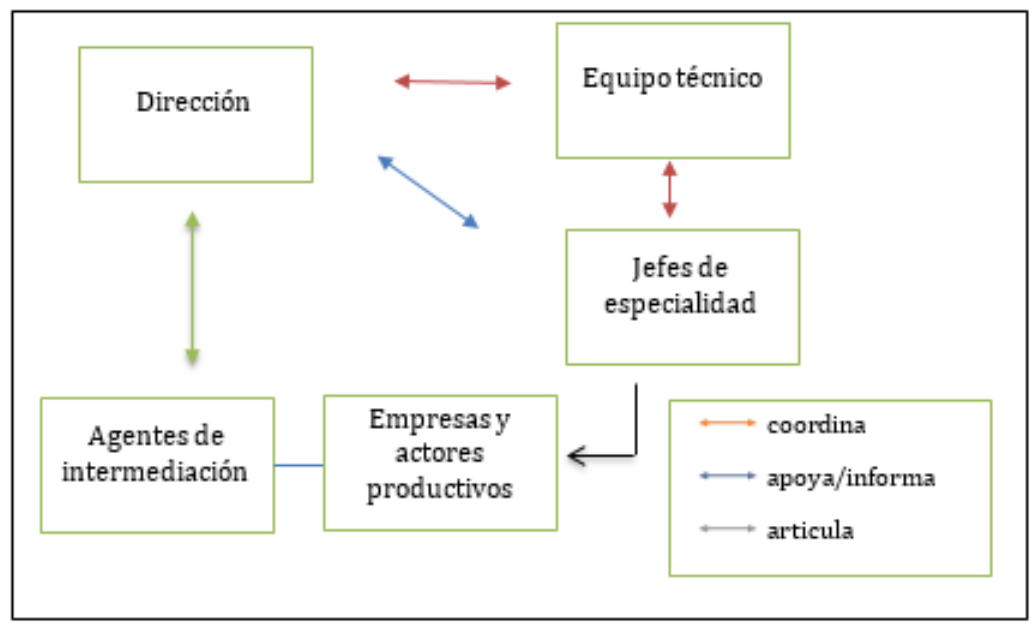

Figura 2. Modelo de articulación y apoyo de la gestión.

Fuente: Elaboración propia.

Finalmente, en este estudio también fue posible identificar un tercer modelo de gestión de la oferta de la EMTP, que corresponde a iniciativas de intermediación institucional para posibilitar una adecuada vinculación con el mundo productivo y el conjunto de organizaciones que pueden ser relevantes para el desarrollo de la oferta formativa institucional. La intermediación externa es una demanda cuando las propias capacidades internas no son suficientes para responder al objetivo de creación, profundización y/o renovación de tales vínculos. Los casos 2 y 6 son particularmente paradigmáticos en esta dirección; por una parte, esta intermediación se reconoce en la figura de un gerente que instala el propio sostenedor para esta tarea (modelo institucional que se encuentra en algunos establecimientos de administración delegada) y, por otra, en la decisión de la Corporación Municipal a cargo de la oferta formativa de la EMTP en una comuna, que contrata a una profesional para favorecer nuevos vínculos y estrategias de articulación público-privadas asociadas a las especialidades de cada establecimiento educacional.

El modelo de intermediación es una demanda altamente sentida en el ámbito municipal, sobre todo debido a la sobrecarga administrativa que implica la gestión directiva en estos establecimientos, por lo que la figura de los Servicios Locales de 
Educación Pública, actualmente en proceso de implementación en el sistema educativo chileno, puede jugar un rol relevante en la tarea de intermediación aquí reseñada.

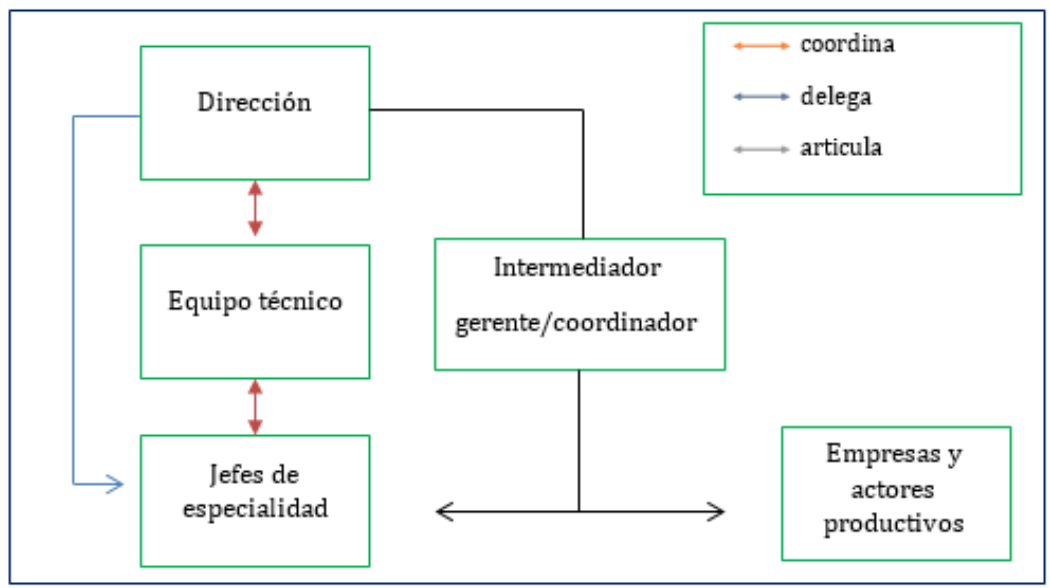

Figura 3. Modelo de intermediación para la gestión.

Fuente: Elaboración propia.

Esta figura o modelo de gestión evidencia una forma de liderazgo complementario al que generalmente ejercen los directivos de los establecimientos educacionales, ya que refiere a una tarea que excede los ámbitos administrativos y pedagógicos los que, por lo general, absorben la mayor parte del tiempo de los líderes escolares. Con todo, es importante destacar que esta función tiene implicancias educativas evidentes; representa un mecanismo muy importante para la revisión y actualización curricular y es el medio fundamental para lograr adecuadas prácticas profesionales para los estudiantes, entendiendo estas últimas como actividades formativas contenidas en el currículo de formación diferenciada.

\section{d. Perspectivas de liderazgo en la gestión de la EMTP}

Como se ha intentado demostrar, este estudio aporta evidencia acerca de la manera en que se gestiona la provisión educativa de la EMTP y, muy particularmente, del modo en que se enfrentan algunos de los nudos problemáticos de este sistema. A diferencia de experiencias formativas de otras latitudes, los directivos de los centros que 
ofrecen EMTP en nuestro país se alejan de la figura de un manager o administrador de una organización, teniendo roles y funciones pedagógicas que les exige un fuerte liderazgo destinado a mejorar resultados educativos en el primer ciclo de la enseñanza media ${ }^{17}$.

Lo anterior puede ser parte de la explicación del fenómeno de liderazgo delegativo analizado aquí y que tiene un efecto de encapsulamiento y/o fragmentación de la gestión de la oferta EMTP en muchos establecimientos educacionales. Para el desarrollo de propuestas formativas específicas de la EMTP (adaptación curricular e innovación), el estudio evidencia una mayor debilidad en el ejercicio de un liderazgo instructivo o aquel destinado al desarrollo de alternativas curriculares y mayor autonomía, tal como sugieren estudios en este campo (por ejemplo, Bouwmans, Runhaar, Wsselink \& Mulder, 2017).

Salvo casos excepcionales de una gestión directiva que dinamiza procesos de alta innovación (como ocurre en el caso 1), la sostenibilidad de una propuesta de formación en el área de educación técnica depende de la creación de equipos de trabajo y lazos de confianza que se sostengan a lo largo del tiempo. Los casos de este estudio son indicativos de que esta es una fortaleza del sistema EMTP en general. Con todo, la calidad de una oferta pertinente y funcional a las necesidades del mundo productivo (tal como lo sugiere Cedefop) requiere de otro tipo de capacidades institucionales que, en muchos casos, son particularmente débiles o fragmentadas. La existencia de una red de intermediación institucional, tal como ocurre en el caso 6, constituye una forma de liderazgo complementario que potencialmente sería de gran relevancia para el sistema de provisión pública en el futuro.

17 Considérese solo este dato: de los 950 establecimientos que imparten la ESTP en Chile, 192 se encuentran en el nivel de insuficiente en la medición de la ACE, y un poco más de 300 tienen calificación medio bajo, existiendo una alta presión en los equipos directivos para el mejoramiento de los resultados escolares. Políticas recientes orientadas a establecimientos ESTP enfatizan en esta dirección (véase, por ejemplo, Política de liceos bicentenarios en https://liceosbicentenario.mineduc.cl/). 


\section{Conclusiones}

Los establecimientos que imparten la educación media técnico profesional deben resolver una serie de nudos específicos de gestión que interrogan acerca de las formas de liderazgo educativo que pueden ponerse en juego para su adecuado enfrentamiento. Como se indicó inicialmente, estos centros educativos están sometidos a las mismas exigencias y formas de evaluación que el resto de los establecimientos que imparten enseñanza media, pero de manera adicional, deben cumplir con las exigencias de la formación para el trabajo en las especialidades que imparten, asumiendo una serie de tareas particulares que incluyen la apertura al entorno productivo y la construcción de una fuerte relación con empresas de los rubros asociados a su oferta formativa. Junto con lo anterior, y tal como se evidencia en las principales fuentes bibliográficas acerca de este ámbito a nivel internacional, uno de los mayores desafíos institucionales es la constante adecuación curricular a los cambios socioproductivos y la forma de organización laboral, con el objetivo de entregar una oferta pertinente y actualizada.

A partir de la reforma curricular del año 1998 (la más importante reforma de esta modalidad en los últimos 50 años), el sistema de EMTP en Chile se caracteriza por una orientación de tipo comprensiva, que busca equilibrar una oferta que articule al mismo tiempo una adecuada formación para el trabajo con el desarrollo de competencias para que sus egresados tengan la posibilidad de continuar estudios superiores, ya sea en el área formativa de su especialidad estudiada, o bien, en otro campo o área temática. Como da cuenta la evidencia recogida en este estudio, este doble objetivo de la EMTP es difícilmente alcanzado por los centros educativos, pues se observan dificultades de gestión institucional que interrogan acerca de las formas de liderazgo educativo que pueden ponerse en juego para un adecuado enfrentamiento de estos desafíos.

A partir del estudio de casos de esta investigación, es posible sostener que, por lo general, se abordan de manera débil algunos de los desafíos estratégicos propios de la EMTP (orientación vocacional y articulación curricular), aunque existen mecanismos institucionales 
para hacerles frente. Estos nudos, muchas veces, no dependen exclusivamente de la gestión de los liceos y constituye un problema del sistema en su conjunto, no obstante, resulta evidente la debilidad de propuestas de innovación en el ámbito de la formación para el trabajo en la gran mayoría de los establecimientos educacionales.

Por otra parte, la relación con el entorno productivo es un problema extendido, y solo es posible observar mecanismos o procedimientos de vinculación satisfactorios cuando existe un soporte institucional del sostenedor para facilitar esta tarea. Donde predomina una forma de liderazgo que recae en los jefes de especialidad, situación que ocurre en la mayoría de los centros, se evidencian dos problemas fundamentales; el primero es que esta forma de liderazgo de la gestión de la especialidad depende de las redes específicas que los propios profesionales tienen o han construido en el tiempo, existiendo diferencias evidentes en un análisis caso a caso, cuestión que puede incidir en formas de segmentación interestablecimientos en un ámbito estratégico para la formación EMTP. El segundo problema de esta forma de liderazgo es que, en sentido estricto, denota una consideración menor de este nudo en los temas directivos, toda vez que en su gran mayoría, los jefes de especialidad o coordinadores TP no participan en los equipos de dirección. De este modo, los problemas propios de la EMTP pueden quedar relegados, ante la preocupación por las cuestiones propias de la formación general, más allá de la definición e identidad particular de los centros en cuestión.

Para el conjunto de establecimientos del sector municipal (más de la mitad de la actual oferta institucional de la EMTP), la existencia de una intermediación por parte del sostenedor representa una interesante alternativa. La evidencia del caso analizado donde opera esta figura de intermediación puede ser de mucha relevancia para futuras políticas, como ocurre con los futuros Servicios Locales de Educación actualmente en proceso de implementación.

Finalmente, es muy importante señalar que una discusión en torno a las modalidades de gestión y ejercicio del liderazgo al interior de la EMTP no puede restarse del debate acerca de los desafíos futuros de la demanda educativa en una sociedad como la 
nuestra. El crecimiento de las aspiraciones de las nuevas generaciones de estudiantes y la mayor tasa de ingreso a la educación superior verificada en los últimos años (y muy particularmente entre estudiantes de la EMTP), constituyen antecedentes relevantes en esta dirección, que pueden poner en entredicho un modelo de diferenciación temprana en la enseñanza media como el actualmente vigente, exigiendo un debate más profundo acerca del rol y el lugar de la educación técnica en relación con lo que se han denominado las competencias del siglo XXI.

\section{Referencias}

Adams, D. \& Gamage, T. (2008). A study of leadership effectiveness in a large VET institution in Australia. International Journal of Educational Management, 22(3), 214-228. https://doi. org/10.1108/09513540810861856

Agencia de Calidad de la Educación, ACE. (2016a). Estudio sobre la calidad educativa en la educación secundaria técnico profesional (EMTP) desde la perspectiva de los actores claves del sistema: fase cualitativa. Recuperado de https://bibliotecadigital.mineduc.cl/handle/20.500.12365/4538

Agencia de Calidad de la Educación, ACE. (2016b). Panorama de la educación secundaria técnico profesional en Chile Recuperado de http://archivos. agenciaeducacion.cl/Panorama_Ed_TP_en_Chile.pdf

Amaral, N., De Diego, M. E., Pagés, C., y Prada, M. F. (2018). Hacia un sistema de formación técnico profesional en Chile: un análisis funcional. Banco Interamericano de Desarrollo, BID (Nota técnica $n^{\circ}$ 1.522). Recuperado de https://publications.iadb.org/es/publicacion/hacia-un-sistema-deformacion-tecnico profesional-de-chile-un-analisis-funcional

Arias, E., Farías, M., González-Velosa, C., Huneeus, C., y Rucci, G. (2015). Educación técnica profesional en Chile. Recuperado de https:// publications.iadb.org/bitstream/handle/11319/6881/Educacion_ tecnico_profesionalChile.PDF

Boateng, C. (2012). Leadership styles and effectiveness of principals of vocational technical institutions in Ghana. American International Journal of Contemporary Research, 2(3), 128-134.

Bouwmans, M., Runhaar, P., Wsselink, R., \& Mulder, M. (2017). Towards distributed leadership in vocational education and training schools: The interplay between formal leaders and team members. Educational Management Administration \& Leadership, 20(10), 1-17 http://dx.doi. org/10.1177/1741143217745877 
Carbone, R., Fuenzalida, C., y Farías J. M. (2017). Definición de estándares de desempeño para equipos directivos técnico profesionales e identificación de factores que facilitan u obstaculizan alcanzarlos. Cuadernos para el desarrollo del liderazgo educativo, $\mathrm{n}^{\circ}$ 5. Santiago de Chile: Centro de Desarrollo del Liderazgo Educativo, CEDLE. Recuperado de http:// cedle.cl/wp-content/uploads/2017/06/Cuaderno-5.pdf

Cariola, L. (1992). Dilemas sobre la restructuración de la educación secundaria. En S. Rittershaussen y J. Scharager (Eds.), Análisis y proyecciones en torno a la educación secundaria y el trabajo (pp. 15-55). Santiago de Chile: Corporación de Promoción Universitaria, CPU.

Cariola, L., Labarca, G., Irigoin, M., Erazo, S., y Fox, E. (1994). La educación secundaria en el mundo: estructura y diseño curricular en diferentes países. Colección de estudios sobre educación secundaria. Santiago de Chile: Programa de Mejoramiento de la Calidad y Equidad de la Educación, Mineduc.

Centro de Estudios de Políticas y Prácticas en Educación, CEPPE. (2017). Servicio de análisis del sistema de administración delegada creado por el DL n 3166 de 1980. Santiago de Chile: Autor.

Comisión Nacional para la Productividad. (2018). Formación de competencias para el trabajo en Chile. Recuperado de https://www. comisiondeproductividad.cl/wp-content/uploads/2018/03/Informe_ Formacion-de_Competencias-para_el_Trabajo.pdf

Comisión para el Estudio de la Formación Técnico Profesional en Chile, Ministerio de Educación de Chile, Mineduc. (2009). Bases para una política de formación técnico profesional en Chile (Informe ejecutivo). Santiago de Chile: Autor. Recuperado de http://educacion2020. cl/documentos/bases-para-una-politica-de-formacion-tecnicoprofesional-en-chile/

Comisión para la Educación de Calidad para Todos. (2016). Construyendo una educación de calidad: un pacto con el futuro de América Latina. Buenos Aires: Fundación Santillana. Recuperado de http://1mlnttzpbhl3wbhhgahbu4ix.wpengine.netdna-cdn.com/wpcontent/uploads/2016/08/Construyendo-una-educación-de-calidadWEB.pdf

Del Campo, G. y Cárcamo, J.C. (1993). Administración moderna de establecimientos de enseñanza técnica. Santiago de Chile: Centro de Investigación y Desarrollo de la Educación, CIDE.

Donoso, S. y Corvalán, O. (2012). Formación técnica y aseguramiento de la calidad: enfoque de desarrollo de competencias. Cuadernos de Pesquisa, 42(146), 612-639. https://doi.org/10.1590/s010015742012000200015 
Educación 2020. (2016). 15 propuestas para cambiar la historia de la educación técnica. Recuperado de http://educacion2020.cl/documentos/15propuestas-para-cambiar-la-historia-de-la-educacion-tecnical

European Centre for the Development of Vocational Training, Cedefop. (2011). Exploring leadership in vocational education and training (Working paper $\mathrm{N}^{\circ} 13$ ) Luxembourg: Publications Office of the European Union. Recuperado de http://www.cedefop.europa.eu/en/publications-andresources/publications/6113

Khatajabor, M., Asnuldahar, M., Sadaadamu, M., \& Buntat, Y. (2012). Sustainable leadership for technical and vocational education and training in developing nations. International Journal of Scientific and Research Publications, 2(8), 1-5. Recuperado de http://www.ijsrp.org/ research-paper-0812/ijsrp-p0892.pdf

Larrañaga, O., Cabezas, G., y Dusaillant, F. (2013). Educación técnico profesional: trayectoria educacional e inserción laboral de una cohorte de alumnos. Santiago de Chile: Programa de las Naciones Unidas para el Desarrollo, PNUD. Recuperado de https://www.undp.org/content/ dam/chile/docs/pobreza/undp_cl_pobreza_informe_completo_ETP.pdf

Maclean, R. \& Pavlova, M. (2013). Vocationalization of secondary and higher education: Pathways to the world of work. Recuperado de https://unevoc. unesco.org/fileadmin/up/2013_epub_revisiting_global_trends_in_ tvet_chapter2.pdf

Martuccelli, D. y Santiago, J. (2017). El desafí sociológico hoy: individuo y retos sociales. CIS no 305. Madrid: Centro de Investigaciones Sociológicas.

Ministerio de Educación de Chile, Mineduc. (2015). Bases curriculares, planes y programas de estudio. Formación diferenciada técnico profesional. Recuperado de http://media.mineduc.cl/wp-content/uploads/ sites/28/2016/04/Cartilla-Curricular-EMTP-1.pdf

Ministerio de Educación de Chile, Mineduc. (2018). Estrategia nacional de formación técnico profesional. Recuperado de http://www. tecnicoprofesional.mineduc.cl/wp-content/uploads/2018/03/ Estrategia-Nacional-de-Formación-Técnico-Profesional.pdf

Miranda, M. (2005). Transformaciones de la educación secundaria técnico profesional. En C. Cox (Ed.), Políticas educacionales en el cambio de siglo: la reforma del sistema escolar en Chile (pp. 375-418). Santiago de Chile: Editorial Universitaria.

Mulder, R., Messmann, G., \& König, C. (2015). Vocational education and training: Researching the relationship between school and work. European Journal of Education, 50(4), 497-512. http://dx.doi. org/10.1111/ejed.12147 
Organización de las Naciones Unidas para la Educación, la Ciencia y la Cultura, Unesco. (2016). La enseñanza y la formación técnico profesional en América Latina y el Caribe: una perspectiva regional hacia 2030. Recuperado de http://unesdoc.unesco.org/images/0026/002607/260709s.pdf

Organización para la Cooperación y el Desarrollo Económicos, OCDE. (2017). Educación en Chile; evaluaciones de politicas nacionales de educación. Santiago de Chile: Fundación SM. Recuperado de http://archivos. agenciaeducacion.cl/Educacion_en_Chile_OCDE_Nov2017.pdf

Özdemir, G. \& Şahin, S. (2016). Development of the technical leadership skills scale in vocational and technical high school administrators: Reliability and validity studies. Journal of Educational Sciences Research, 6(1), 135-152.

Park, J. -H. \& Jeong, D. (2013). School reforms, principal leadership, and teacher resistance: Evidence. Asia Pacific Journal of Education, 33(1), 34-52. http://dx.doi.org/10.1080/02188791.2012.756392

Polesel, J., Klatt, M., Blake, D., \& Starr, K. (2017). Understanding the nature of school partnerships with business in delivery of vocational programmers in schools in Australia. Journal of Education and Work, 30(3), 283-298, https://doi.org/10.1080/13639080.2016.1165344

Raffe, D. (2007). Making haste slowly: The evolution of a unified qualifications framework in Scotland. European Journal of Education, 42(4), 485-502. https://doi.org/10.1111/j.1465-3435.2007.00322.x

Sepúlveda, L. (2016). Trayectorias educativo-laborales de jóvenes estudiantes de educación técnica en Chile: ¿tiene sentido un sistema de formación para el trabajo en la educación secundaria? Revista Páginas de Educación, 9(2), 189-212. https://doi.org/10.22235/pe.v9i2.1297

Sepúlveda, L., Ugalde, P., y Campos, F. (2011). La enseñanza media técnico profesional en Chile: orientaciones actuales desde la perspectiva de sus actores. En Centro de Estudios, Mineduc (Ed.), Evidencias para políticas públicas en educación: selección de investigaciones tercer concurso FONIDE. Tomo I (pp. 15-52). Santiago de Chile: Autor.

Sepúlveda, L. y Valdebenito, M. J. (2014). ¿Las cosas claras? Aspiraciones de futuro y proyecto educativo laboral de jóvenes estudiantes secundarios. Estudios Pedagógicos (Valdivia), 40(1), 243-261. http:// dx.doi.org/10.4067/S0718-07052014000100015

Sevilla, P. (2011). Educación técnica profesional en Chile: antecedentes y claves de diagnóstico. Santiago de Chile: Centro de Estudios Ministerio de Educación. Recuperado de http://www.tecnicoprofesional.mineduc. cl/descargas/material-de-apoyo-y-consulta/ 
Sevilla, P. (2017). Panorama de la educación técnico profesional en América Latina y el Caribe. Serie Políticas Sociales $n^{\circ} 222$. Santiago de Chile: CEPAL y Ministerio de Asuntos Exteriores de Noruega. Recuperado de https://repositorio.cepal.org/bitstream/handle/11362/40920/1/ S1601350_es.pdf

Sevilla, P. y Sepúlveda, L. (2016). Selección en liceos polivalentes. Análisis de los mecanismos de adscripción de estudiantes a la modalidad científicohumanista o técnico-profesional. Recuperado de https://centroestudios. mineduc.cl/wpcontent/uploads/sites/100/2017/06/REVISTA EPE_ N3.pdf

Simons, M. \& Harris, R. (2014). Educational leadership in Australian private VET organizations: How is it understood and enacted? Research in Post-Compulsory Education, 19(3), 245-260. http://dx.doi.org/10.108 0/13596748.2014.920562

Stake, R. (2010). Investigación con estudio de casos. Madrid: Morata.

Stake, R. (2013). Estudios de casos cualitativos. En N. Denzin e Y. Licoln (Coords.), Las estrategias de investigación cualitativa. Manual de investigación cualitativa. Volumen III. Barcelona: Gedisa.

Stern, D. (2009). Expanding policy options for educating teenagers. The Future of Children, 19(1), 211-239. https://doi.org/10.1353/foc.0.0027

Recibido: 13/06/2019

Aceptado: 17/10/2019 